\title{
Les ménages agricoles aux États-Unis
}

Participation au travail, prise de décision et influence du contrôle des actifs agricoles

Agricultural Households in the États-Unis. Participation to Labor, Decisionmaking and the Effects of Farm Asset Control

Jill L. Findeis, Hema Swaminathan et Anuja Jayaraman

Traducteur : Catherine Moreddu

\section{OpenEdition}

Journals

\section{Édition électronique}

URL : http://journals.openedition.org/economierurale/112

DOI : 10.4000/economierurale. 112

ISSN : 2105-2581

Éditeur

Société Française d'Économie Rurale (SFER)

Édition imprimée

Date de publication : 30 décembre 2005

Pagination : 44-62

ISSN : 0013-0559

Référence électronique

Jill L. Findeis, Hema Swaminathan et Anuja Jayaraman, «Les ménages agricoles aux États-Unis », Économie rurale [En ligne], 289-290 | Septembre-décembre 2005, mis en ligne le 02 mars 2009, consulté le 21 avril 2019. URL : http://journals.openedition.org/economierurale/112 ; DOI : 10.4000/ economierurale. 112

Ce document a été généré automatiquement le 21 avril 2019.

(C) Tous droits réservés 


\section{Les ménages agricoles aux États-Unis}

Participation au travail, prise de décision et influence du contrôle des actifs agricoles

Agricultural Households in the États-Unis. Participation to Labor, Decision-

making and the Effects of Farm Asset Control

Jill L. Findeis, Hema Swaminathan et Anuja Jayaraman

Traduction : Catherine Moreddu

\section{NOTE DE L'ÉDITEUR}

Traduction de Catherine Moreddu, OCDE

\section{NOTE DE L'AUTEUR}

Les auteurs souhaitent remercier les autres membres du Groupe de recherche "The Women on U.S. Farms Initiative Research Group", notamment Carolyn Sachs, Fern Willits, Atsuko Nonoyama, Amy Trauger, Irene Tanzo et Natalie Jolly. Ils sont reconnaissants de la générosité de la défunte Rachel Rosenfeld qui a fourni les données de l'enquête de 1980. Ces travaux de recherche font partie d'un projet financé par le « National Research Initiative (NRI) Competitive Grant No AG990181 » du Département agricole des États-Unis (USDA) et par un accord de coopération (No 43-3AEL-80070) avec le Service de recherche économique de l'USDA. Les auteurs remercient les reviewers anonymes pour leurs commentaires et suggestions.

\section{Introduction}

1 Les ménages agricoles présentent une certaine complexité interne qui est importante pour les politiques. Partout dans le monde, les ménages agricoles sont dépendants de 
multiples sources de revenu apportées par les individus qui composent le ménage. Les membres du ménage diffèrent en termes de productivité, leurs préférences en matière d'emploi peuvent varier et ils sont affectés diversement par les normes sociales et même par les restrictions liées au droit du travail. La pluriactivité est fréquente et les choix en matière d'emploi au sein du ménage dépendent d'un ensemble complexe de relations dans lesquelles les individus parfois coopèrent, parfois ne coopèrent pas. Même sur une exploitation, les membres du ménage ont souvent des rôles différents selon leur sexe, leur statut ou leur âge. Ces rôles peuvent influencer la nature et le montant de la production destinée à l'autoconsommation ainsi que la nature et le montant du chiffre d'affaires agricole. Ils influent également sur la façon dont les bénéfices, les biens autoconsommés et le revenu agricole sont répartis. Les choix en matière de dépenses varient au sein des ménages selon des préférences différentes pour les hommes et les femmes et qui évoluent au cours du cycle de vie. Dans les pays en développement où les choix en matière d'alimentation, d'éducation et de santé ont une incidence marquée sur la vie des membres du ménage, en particulier les enfants, les décisions du ménage peuvent avoir une grande importance (Doss, 1996; Quisumbing et de La Brière, 2000 ; Swaminathan et Findeis, 2003). Il est important de reconnaître qu'il est indispensable de considérer les différences au sein des ménages pour comprendre les choix économiques et le bien-être des individus car elles donnent accès à la « boîte noire » que constitue un ménage. C'est particulièrement vrai en ce qui concerne les politiques, car de nombreuses interventions ont pour objectif explicite l'amélioration du bien-être du ménage, et donc des individus qui le composent. Même lorsque l'objectif n'est pas spécifiquement centré sur les ménages ou les individus, ils sont souvent la cible prétendue.

2 Cet article porte plus particulièrement sur la participation au travail et à la prise de décision au sein de l'exploitation des femmes agricultrices aux États-Unis. Il explore tout d'abord brièvement l'importance, au plan historique, des entreprises-exploitations agricoles dans les pays développés. À l'aide de données d'une enquête nationale sur les agricultrices aux États-Unis, menée en 2001 à Penn State University en collaboration avec le Service de recherche économique (Economic Research Service, ERS) et le Service National de la statistique agricole [National Agricultural Statistics Service, NASS du département de l'agriculture aux États-Unis (USDA)], l'évolution de l'emploi non agricole, du revenu et du patrimoine des exploitations agricoles au États-Unis est ensuite examinée, suivie de celle de la participation des femmes à des tâches spécifiques et aux décisions concernant l'exploitation. Des comparaisons sont effectuées entre les données de 2001 et celles collectées en 1980 par Rosenfeld (1985) sur les agricultrices et les ménages agricoles.

3 Les analyses descriptives sont suivies d'une présentation de modèles qui examinent les déterminants de l'emploi non agricole et de la prise de décision par les agricultrices des États-Unis. L'attention se porte sur les agricultrices du fait du changement très net de comportement, en termes d'emploi, de cette population depuis au moins les deux dernières décennies. Les effets au sein du ménage sont considérés pour mieux comprendre les décisions en matière de répartition du travail qui affectent les revenus totaux de l'exploitation agricole et la participation aux décisions concernant l'exploitation agricole. Le rôle du type de transmission de l'exploitation entre génération - selon que l'exploitation vient de la famille de l'homme ou de celle de la femme - sur les décisions en matière d'emploi et la prise de décision relative à l'exploitation agricole est d'une importance toute particulière. L'intérêt pour cette question vient de la part 
croissante des femmes qui héritent maintenant des exploitations de leur famille aux États-Unis.

\section{Perspective historique Les ménages agricoles dans les pays développés}

Depuis le milieu des années 1950, les travaux d'économie et d'économie agricole sur les exploitations agricoles dans les pays développés ont porté dans une large mesure sur des questions directement liées à la profitabilité de l'entreprise - réaction de l'offre, efficacité de l'exploitation, risques et incertitudes, prise de décision en cas de production jointe et de produits multiples, et sur les effets de la politique agricole, parmi tant d'autres questions pertinentes. Ces travaux différaient sur le fonds de ceux qui les avaient précédés. Premièrement, ils étaient plus techniques et moins descriptifs. Ils s'appuyaient d'une part sur les avancées importantes réalisées dans le domaine de l'économétrie, de la recherche sur les opérations et du calcul mathématique. D'autre part, les outils méthodologiques utilisés étaient plus appropriés à l'analyse des entreprises agricoles individuelles et aux systèmes d'entreprises qu'à celle de la situation de «l'exploitation », c'est-à-dire de l'unité entreprise-ménage agricole, en termes de revenu total et d'actifs. C'est dans les travaux sur les pays en développement en économie et économie agricole que l'unité entreprise-ménage agricole a été la plus étudiée.

5 Le changement actuel vient du fait que la réforme des politiques agricole dans de nombreux pays développés a conduit à s'intéresser de nouveau à l'entreprise-ménage agricole et aux comportements au sein du ménage (OCDE, 2001) ${ }^{1}$. La hausse de la part des sources de revenu non agricoles indépendantes des activités sur l'exploitations dans le revenu des ménages agricoles et de la diversification des actifs de l'entreprise-ménage agricole a permis de souligner la nécessiter d'adopter une perspective plus large comprenant l'entreprise-ménage agricole.

6 Le recours accru des ménages agricoles aux revenus et aux actifs non agricoles remet en question le rôle des politiques vis-à-vis des exploitations - une politique agricole est-elle nécessaire pour soutenir le niveau de revenu des entreprises-ménages agricoles ou bien l'objectif sous-jacent est-il différent? La grande question de l'utilisation des terres en agriculture plaide également pour une perspective en termes de ménages. Dans le passé, l'évolution de la profitabilité des exploitations a influencé l'utilisation des terres mais dans certaines régions des États-Unis, cette relation est probablement plus faible qu'auparavant. Du fait de la hausse significative de la dépendance des ménages agricoles envers des revenus non agricoles, il est probable que l'utilisation des terres dans les zones les plus vulnérables, en termes de perte de foncier agricole au profit de développements industriels et/ou résidentiels, dépendent moins de la profitabilité de l'exploitation que de la capacité de l'entreprise- ménage agricole à se constituer un « portefeuille » de revenus lui permettant de conserver l'exploitation. En effet, les sorties du secteur pourraient dépendre davantage du revenu total de l'entreprise-ménage agricole que de la simple profitabilité de l'exploitation. Enfin, la volonté des enfants de reprendre l'exploitation pourrait être fortement influencée par leur capacité à trouver des emplois non agricoles qui procurent un niveau raisonnable de revenu pour l'entreprise-ménage agricole.

7 L'intérêt pour les ménages résulte également des avancées simultanées enregistrées dans les cadres théoriques utilisés pour analyser les ménages. Les travaux classiques de Gary 
Becker et Jacob Mincer ont attiré l'attention sur les ménages et les familles. Le modèle unitaire de Becker (1991), largement appliqué aux ménages des pays en développement (Singh et al, 1986), est un point de départ utile et continu à être fréquemment et utilement employé pour comprendre les productions des ménages. Cependant, comme le font remarquer Udry et al (1995), les faits empiriques contredisent le concept que 'les ménages agissent comme des individus'. Au contraire, il est plus probable que d'importantes interactions agissent au sein des ménages et qu'elles affectent les résultats observés différences de structure des dépenses, variations de l'offre de travail et des choix de production agricole, entre autres décisions. Des points de vue différents concernant les interactions et les comportements au sein des ménages se sont exprimés (Kooreman et Wunderlink, 1997), notamment les modèles de ménages collectifs associés à Chiappori (1988, 1992, par exemple) et les modèles de ménages de négociation de Manser et Brown (1980) et McElroy et Horney (1981). Ces cadres sont importants dans les travaux publiés sur les pays en développement (Schultz, 1999; Quisumbing, 2003), et ils ont été récemment appliqués aux ménages agricoles des pays développés (Findeis et Swaminathan, 2002 ; Keyzer et Phimister, 2003). En ce qui concerne les ménages agricoles, les questions relatives à la répartition du travail au sein du ménage et au transfert international des actifs sont importantes (Pesquin et al, 1999; Kimhi et Nachlieli, 2001 ; Errington, 2002, par exemple).

Des évolutions importantes ont été observées au sein des ménages agricoles au cours des deux dernières décennies ou plus - par exemple, des hausses de l'âge moyen de la population agricole, des baisses de fertilité, davantage de femmes chef d'exploitation, et des différences en terme d'héritage, entre autres 'mutations'. Cet article se concentre sur les 'mutations' qui affectent la relation entre les unités entreprise-ménage agricoles et les marchés de l'emploi extérieurs (hors de l'exploitation ou non agricole), en particulier parmi les agricultrices, et qui ont enregistré des changements très importants. Cet article s'intéresse aussi aux effets internes aux ménages et à l'influence du mode de transmission intergénérationnelle de l'exploitation d'abord sur les choix des agricultrices en matière d'emploi ensuite sur leur participation à la prise de décision concernant l'exploitation agricole.

\section{Données, modèle théorique et méthodes d'estimation}

Cet article fait appel à des données sur les femmes en agriculture et sur les ménages agricoles collectées en 2001 par des chercheurs de Penn State University pour évaluer les évolutions observées et les effets au sein des ménages. Il est divisé en deux sections. Premièrement, les données de l'enquête de 2001 sont comparées aux résultats de la dernière enquête précédente sur les femmes en agriculture et les ménages agricoles aux États-Unis conduite par Rosenfeld en 1980 (Rosenfeld, op. cit.). Entre les enquêtes de Penn State et de Rosenfeld, certaines questions sont totalement comparables, ce qui permet d'analyser les changements. Les données de 2001 sont ensuite utilisées pour examiner les déterminants de l'emploi non agricole parmi les femmes agricultrices et l'influence de la transmission de l'exploitation entre les générations - selon que l'exploitation vient de la famille de l'homme ou de celle de la femme - sur les comportements en terme d'emploi et de prise de décision. Cette variable est rarement considérée dans les modèles de répartition du travail entre activités agricoles et non agricoles, même si elle influence probablement les décisions prises par le ménage et par les individus qui le composent. 
Nous avançons que l'origine de la transmission intergénérationnelle affecte la mesure dans laquelle les individus décident de s'engager dans des activités extérieures non agricoles et d'exercer une influence sur les décisions relatives à l'exploitation agricole.

\section{Données utilisées}

L'enquête de Penn State University sur les femmes agricultrices aux États-Unis a été réalisée en avril 2001, par entretien téléphonique avec des agricultrices sélectionnées selon un cadre d'échantillonnage utilisé par le Département de l'agriculture (USDA). L'enquête nationale de 2001 a été menée par Penn State University en collaboration avec le NASS et ERS. À l'aide d'un échantillonnage aléatoire, un total de 2661 observations a été retenu. On a demandé à chaque participante de répondre à des questions sur :

1. son rôle dans la prise de décisions relatives à l'exploitation agricole,

2. sa participation à des tâches agricoles spécifiques et l'utilisation de pratiques agricoles durables,

3. ses activités professionnelles non agricoles, y compris dans une profession indépendante non agricole,

4. sa détention et acquisition par héritage d'actifs tels que des terres agricoles,

5. sa participation à des organisations agricoles et communautaires et ses éventuelles responsabilités dans ces organisations,

6. les caractéristiques démographiques de son ménage et des membres qui le composent,

7. les caractéristiques de l'exploitation agricole.

11 Les données indiquent également le Comté de résidence, ce qui permet d'y apparier des informations sur les marchés de l'emploi non agricole. Lorsque l'enquête a été terminée, les données ont été associées par Comté de résidence aux neuf régions de production agricole différenciées par l'USDA : Heartland, Prairie Gateway, Northern Crescent, Northern Great Plains, Eastern Uplands, Southern Seaboard, Fruitful Rim, Basin and Range, et Mississippi Portal. Au total, 2444 ménages agricoles ont pu être appariés et forment la base de données de 2001.

Pour l'ensemble de l'échantillon, des données ont également été recueillies sur les activités professionnelles et les tâches du conjoint/compagnon (si disponible). Bien que les données descriptives correspondent à l'ensemble des ménages agricoles qui ont répondu à l'enquête nationale de Penn State University, les estimations empiriques utilisées dans cet article se limitent aux ménages dont les deux conjoints/compagnons étaient présents. Cela réduit quelque peu le nombre d'observations, mais une grande majorité d'entre elles ont été retenues.

\section{Modèle théorique}

13 Le modèle théorique utilisé pour comprendre les choix relatifs à l'emploi non agricole dans le contexte des États-Unis est le modèle du ménage agricole bien connu. Cependant, comme l'accent de ces travaux porte sur les effets internes au ménage, le cadre de négociation au sein du ménage (Manser et Brown, 1980 ; McElroy et Horney, 1981) est utilisé pour explorer les interactions et influences internes. Cette approche a été employée pour comprendre les résultats concernant les femmes dans les pays en développement (Doss, 1996; Schultz, 1999). Elle se prête à l'identification des 
déterminants de la participation des femmes au travail (formel) et à la prise de décisions qui affectent la profitabilité et l'avenir de l'exploitation agricole. La participation à la prise de décisions, par exemple, peut être considérée comme un jeu à deux personnes, dans lequel le pouvoir au sein du ménage affecte les points de rupture, ce qui correspond à un équilibre négocié de Nash (McElroy et Horney, op. cit.; Manser et Brown, op. cit.). Le modèle unitaire (Becker, 1991) ne représente pas explicitement le processus de prise de décision du ménage ni comment est déterminée la répartition de la consommation et du bien-être entre ses membres, ce qui ne permet pas de comprendre clairement les répartitions au sein du ménage et ses incidences en termes de bien-être (Schultz, op. cit.). Ces problèmes posés par le modèle unitaire ont favorisé le développement de modèles alternatifs moins restrictifs qui ont traité de manière explicite la question des préférences individuelles et de l'existence de preneurs de décision multiples au sein du ménage (McElroy et Horney, op. cit.).

Le concept de pouvoir de négociation joue un rôle crucial dans le modèle de négociation de Nash. Le modèle prédit que le processus de répartition du travail et ses résultats va refléter le pouvoir de négociation ou 'l'influence' de l'individu (Quisumbing et de La Brière, 2000). Cependant, les facteurs qui jouent sur la répartition du pouvoir au sein du ménage (dans ce cas entre les conjoints/compagnons) ne devraient pas être déterminés de façon endogène comme l'est la répartition du temps (Schultz, 1999). En ce qui concerne les décisions relatives à l'exploitation agricole, il est probable que l'origine de cette dernière (c'est-à-dire si elle est transmise par la famille de la femme ou celle de l'homme) va jouer sur les résultats, en particulier ceux qui concernent l'exploitation. Les données sur la transmission des exploitations agricoles (transmise par la famille de l'homme, de la femme ou achat sans lien familial) sont disponibles dans les données de 2001. Les capacités en capital humain de la femme agricultrice et de son conjoint/ compagnon pourraient affecter cette relation et elles sont considérées comme des variables exogènes dans les estimations. Si l'on suppose que les couples d'agriculteurs aux États-Unis ont une relation de coopération, le modèle théorique peut s'écrire comme suit :

\begin{tabular}{|l|l|}
\hline $\operatorname{MaxN}-\left[\mathrm{U}^{\mathrm{m}}\left(\mathrm{C}, \mathrm{L}^{\mathrm{m}}\right)-\mathrm{V}^{\mathrm{m}}\left(\mathrm{P}_{\mathrm{e}}, \mathrm{P}_{\mathrm{q}}, \mathrm{P}_{\mathrm{x}}, \mathrm{w}^{\mathrm{m}}, \mathrm{I}^{\mathrm{m}} ; \mathrm{\alpha}^{\mathrm{m}}\right)\right]^{*}$ & $(1)$ \\
\hline$\left[\mathrm{U}^{\mathrm{f}}(\mathrm{C}, \mathrm{Lf})-\mathrm{V}^{\mathrm{f}}\left(\mathrm{P}_{\mathrm{e}}, \mathrm{P}_{\mathrm{q}}, \mathrm{P}_{\mathrm{x}}, \mathrm{w}^{\mathrm{f}}, \mathrm{I}^{\mathrm{f}} ; \alpha^{\mathrm{f}}\right)\right]$ & \\
\hline
\end{tabular}

\begin{tabular}{|l|l|}
\hline$Q=\left(F^{m}, F^{f}, X ; A, \Phi, \Theta\right)$ & $(2)$ \\
\hline$P_{c} C=P_{q} Q-P_{x} X+W^{m} M^{m}+W^{f} M^{f}+I^{m}+I^{f}$ & $(3)$ \\
\hline $\mathrm{T}^{i}=L^{i}+F^{i}+M^{i}, i=f, m$ & $(4)$ \\
\hline$F^{i} \geq 0, i=f, m$ & $(5)$ \\
\hline$M^{i} \geq 0, i=f, m$ & $(6)$ \\
\hline
\end{tabular}


16

et $\mathrm{f}$ sont deux membres du ménage ; $\mathrm{U}^{\mathrm{i}}$ représente la fonction d'utilité de l'individu et $\mathrm{V}^{\mathrm{i}}$ est le point de rupture de l'individu i. Le vecteur $\mathrm{C}$ est un vecteur de la consommation de biens incluant un bien d'intérêt public partagé. X représente l'intrant variable de la production de l'exploitation et $Q$ cette production ; A représente la quantité fixe de terres, $\Phi$ est le vecteur des caractéristiques spécifiques à l'exploitation (par exemple les précipitations et le type de sol), et $\Theta$ représente un vecteur des caractéristiques du ménage. Les prix des biens de consommation, les intrants variables et des produits agricoles sont $\mathrm{P}_{\mathrm{c}}, \mathrm{P}_{\mathrm{x}}$, et $\mathrm{P}_{\mathrm{q}}$, respectivement. Le revenu autre que celui $\mathrm{du}$ travail de l'individu i est $\mathrm{I}^{\mathrm{i}}$. $\mathrm{T}^{\mathrm{i}}$ est le total du temps disponible de l'individu i et il est alloué aux loisirs $\left(\mathrm{L}_{\mathrm{i}}\right)$, à la production agricole $\left(\mathrm{F}^{\mathrm{i}}\right)$ et au travail non agricole $\left(\mathrm{M}^{\mathrm{i}}\right)$, au salaire offert de $w^{\mathrm{i}}$. Enfin, les $\alpha^{\mathrm{i}}$ représentent les 'paramètres environnementaux extérieurs au ménage' ou PEP. Les $V^{i}$ sont les points de rupture des individus et représentent l'utilité que chacun recevrait en dehors de l'accord. Les $\alpha^{\mathrm{i}}$ ou PEP introduits par McElroy (1990) sont interprétés comme les translateurs des points de rupture. Des exemples de PEP seraient les 'paramètres qui décrivent les marchés du mariage, les paramètres qui caractérisent la structure juridique du mariage et $d u$ divorce et les paramètres qui caractérisent les impôts et les transferts publics ou privés qui dépendent du statut marital ou familial' (McElroy, op. cit.). Les ménages résolvent le problème de négociation de Nash en maximisant la fonction objectif de Nash sous contrainte. La participation à la prise de décision peut également être évaluée à l'aide de ce modèle.

\section{Méthode d'estimation}

Les données de l'enquête de Penn State de 2001 sont tout d'abord comparées à celles de Rosenfeld de 1980. L'évolution au cours de la période qui s'étend entre les deux enquêtes de la participation à l'emploi non agricole, des revenus d'activité, des avantages accordés par l'employeur, du patrimoine, des rôles dans l'emploi agricole et de la prise de décisions concernant l'exploitation est examinée.

Des modèles multivariés des décisions relatives à l'emploi sont ensuite estimés, suivis par des modèles de la participation des femmes agricultrices aux États-Unis aux décisions concernant l'exploitation. L'estimation se fait en deux étapes. Il faut tout d'abord déterminer si les décisions relatives au travail non agricole de la femme et de son conjoint/compagnon sont corrélées ('jointes'). Pour commencer, des modèles probit bivariés qui considèrent la jointure des décisions relatives au travail non agricole sont estimés. Si la jointure n'est pas observée, des modèles logit multinomiaux sont alors estimés séparément pour les agricultrices, les catégories de statut du logit multinomial étant les caractéristiques primaires de l'emploi comme la pluriactivité sur et en dehors de l'exploitation. En revanche, en cas de jointure, des modèles logit multinomiaux sont estimés pour les agricultrices, compte tenu des décisions relatives à l'emploi non agricole de leur conjoint/compagnon. C'est-à-dire que les modèles pour les femmes sont estimés selon que leur conjoint/compagnon a un emploi non agricole ou pas. Cette structure est calculable car la moitié environ des femmes agricultrices de l'enquête ont un emploi non agricole et il en est de même pour les conjoints/compagnons.

La variable endogène de "participation à l'emploi non agricole » est mesurée par la participation à des activités non agricoles de la femme ou de son conjoint/compagnon au cours de l'année précédente. Les résultats de l'enquête montrent que presque toutes les femmes agricultrices aux États-unis consacrent une partie de leur temps à travailler sur 
l'exploitation, mais cette participation varie beaucoup dans l'échantillon. Il est clair que certaines femmes sont très impliquées dans les travaux de la ferme, tandis que d'autres ont un rôle beaucoup plus modeste et consacrent d'avantage de temps au travail hors de l'exploitation. La variable représentant la participation au travail agricole est donc fondée sur la réponse des agricultrices interrogées sur leur participation déclarée aux activités de l'exploitation. Les femmes qui ont répondu à l'enquête selon laquelle elles se considéraient comme "le chef d'exploitation", «des partenaires à temps complet » ou "des managers de l'entreprise» ont été définies comme participant aux activités agricoles. Les femmes qui se sont classées elles-mêmes comme des « aides agricoles » ou celles qui n'ont déclaré "aucune participation directe» sur l'exploitation ont été considérées comme n'étant pas impliquées dans les travaux de l'exploitation. Enfin, les femmes qui ont déclaré être impliquées dans des activités à la fois sur et en dehors de l'exploitation ont été classées comme pluriactives. Très peu de femmes se sont retrouvées exclues de l'une ou l'autre de ces catégories. Les variables exogènes incluent:

1. des variables binaires indiquant si l'exploitation provient de la famille de l'homme ou celle de la femme,

2. les caractéristiques des individus censées influencer les décisions relatives au travail (par exemple la dotation en capital humain),

3. les caractéristiques du ménage (par exemple la présence d'enfants de catégories d'âge différents qui reflètent les besoins en supervision par des adultes ou leur capacité à se substituer au travail des parents sur la ferme),

4. des variables concernant l'exploitation qui peuvent affecter les décisions en matière d'emploi. Ces dernières variables comprenaient la part des grandes cultures dans le chiffre d'affaires agricole, le recours à des contrats, et la situation géographique de l'exploitation dans les neuf zones de production agricole définies par l'USDA.

Le temps de travail agricole a été utilisé dans de nombreuses études comme la mesure de l'implication sur l'exploitation à la fois des femmes et des hommes. Une autre mesure est le degré de leur participation à la prise de décision concernant l'exploitation. La dernière section de cet article examine la participation des femmes aux décisions concernant l'exploitation, l'accent étant toujours mis sur les effets de la transmission intergénérationnelle de l'exploitation. Les décisions concernant les terres agricoles, l'achat d'équipements agricoles importants, l'adoption de nouvelles méthodes de production et d'innovations sur l'exploitation et d'autres grandes décisions concernant l'exploitation sont évaluées.

\section{Évolution des rôles dans l'emploi, des revenus d'activité et du patrimoine}

\section{Participation à la main-d'œuvre}

21 Aux États-Unis, l'emploi non agricole des agriculteurs a été remarquablement stable ces dernières années. Parmi les agriculteurs de l'enquête de 2001, 53 \% travaillaient en dehors de l'exploitation l'année précédente, contre $48 \%$ des agriculteurs questionnés par Rosenfeld en 1980 (Rosenfeld, 1985) ${ }^{2}$. Étant donné que la population agricole, tout comme la population américaine en général, compte de plus en plus de retraités, ceci implique qu'une proportion encore plus grande d'hommes en âge de travailler a un emploi non agricole. Si on limite l'échantillon de 2001 aux hommes en âge de travailler (18-64 ans 
inclus), la part des agriculteurs qui ont également un emploi non agricole augmente pour atteindre $57 \%$. Ces données sont conformes à celles du recensement de l'agriculture de 2002 pour les États-Unis. Selon le recensement de l'agriculture de 2002, $55 \%$ des chefs d'exploitation (en grande majorité des hommes) avaient un autre emploi en dehors de leur acticité agricole et $71 \%$ de ceux-ci consacraient 200 jours par an ou davantage à cette autre activité. Ces chiffres sont quelques peu inférieurs aux résultats du recensement de l'agriculture de 1982 dans lequel $58 \%$ des chefs d'exploitation déclaraient travailler en dehors de l'exploitation. Il est cependant important de noter que les recensements de l'agriculture aux États-Unis font apparaître une continuité remarquable des taux de participation à d'autres emplois des chefs d'exploitation au cours des trois dernières décennies ${ }^{3}$.

En revanche, une part croissante des femmes agricultrices, aux États-Unis comme dans de nombreux pays développés, a un emploi salarié en dehors de l'entreprise-exploitation. Cet emploi n'est souvent pas à très proche du lieu de l'exploitation (moins de $5 \%$ des femmes de l'étude de 2001 travaillaient comme employées à domicile). Cette tendance suit celle observée pour les femmes dans l'ensemble de la population - là encore à la fois aux États-Unis comme dans de nombreux pays développés - qui participent de plus en plus à l'emploi salarié formel et au travail indépendant depuis le milieu des années 1960 . Selon l'enquête nationale de Penn State pour 2001, 52 \% des femmes agricultrices aux États-Unis obtenaient des revenus d'un emploi non lié à l'exploitation, contre $37 \%$ dans l'étude de Rosenfeld pour 1979 (Rosenfeld, 1985). En outre, en 2000, parmi les femmes en âge de travailler, ce pourcentage est même plus élevé - $62 \%$ ont un emploi non agricole. En comparaison, le taux de participation générale à la main d'œuvre se montait à $72 \%$ parmi les femmes américaines avec enfants de moins de 18 ans en 2002 (US Department of Labor, 2003b). Parmi les femmes agricultrices ayant un emploi non agricole, $63 \%$ travaillent tout au long de l'année (50-52 semaines) et $80 \% 40$ semaines par an ou plus, ce qui correspond à un emploi lié à l'école (institutrice, conductrice de bus scolaire, assistante de cantine).

La participation à l'emploi non agricole dépend, bien entendu, du stade de la vie, de la taille de l'exploitation et de la situation géographique de l'entreprise-ménage agricole. Les études ont généralement montré que la participation au travail non agricole est la plus élevée à l'âge moyen. Pour les hommes, les taux les plus élevés sont observés de la moitié de la quarantaine au milieu de la cinquantaine. Pour les femmes, les résultats diffèrent selon l'étude et l'époque à laquelle elle a été menée. Dans les premières études, les taux les plus élevés se rencontraient parmi les jeunes femmes, ce qui traduit sans doute l'entrée dans la main-d'œuvre des femmes plus jeunes dans les années 1970. Alors que cette cohorte avançait en âge, les études suivantes ont présenté des taux plus élevés pour le groupe d'âge moyen. La taille de l'exploitation est inversement corrélée aux taux de participation à l'emploi non agricole, en particulier pour les agriculteurs. Ce résultat n'a pas changé de manière significative au cours des trois dernières décennies. Les taux de participation des femmes agricultrices ont cependant présenté un lien plus faible avec la taille de l'exploitation, tout du moins aux États-Unis.

Des différences importantes peuvent être observées parmi les taux de participation des hommes à l'emploi non agricole selon la situation géographique, ainsi que le montrent les différences entre régions de production agricole définies par ERS/USDA. Les taux de participation les plus élevés parmi les agriculteurs en âge de travailler se trouvent dans les régions Fruitful Rim (71 \%) et Eastern Uplands (67\%). Dans la région du Northern Crescent 
qui se caractérise par l'élevage laitier, ce taux est de $58 \%$. Les taux de participation les plus bas pour les agriculteurs en âge de travailler se trouvent dans les régions Northern Great Plains (35\%), Basin and Range (43\%) et Mississippi Portal (39\%). Les régions de production agricole de Northern Great Plains et Basin and Range se caractérisent par de grandes exploitations ou ranchs et un plus grand éloignement des emplois non agricoles (plus rares). L'idée que l'emploi non agricole est un phénomène limité est fausse - par exemple, plus de la moitié des hommes en âge de travailler des exploitations agricoles des régions du Heartland et Prairie Gateway travaillent en dehors de l'exploitation au moins une partie de l'année.

En ce qui concerne les femmes, on observe moins de différences entre régions de production. Là encore, le pourcentage de femmes agricultrices en âge de travailler occupant des emplois non agricoles est le plus faible dans la région Basin and Range, à faible densité de population et caractérisée par des exploitations de très grande taille en nombre d'hectares. Mais même dans cette région, plus de la moitié $(52 \%)$ des femmes agricultrices ont un emploi non agricole. Alors qu'on devrait s'attendre à trouver (et c'est souvent ce qui est avancé) les taux de participation les plus faibles dans la région centrale des États-Unis où la densité de population est la plus faible, les exploitations les plus grandes et l'éloignement des emplois le plus fort, ce n'est pas le cas. Dans la région du Heartland, $67 \%$ des femmes agricultrices en âge de travailler ont un emploi en dehors de l'exploitation. Elle est suivie des régions Prairie Gateway (65\%) et Northern Great Plains (64\%). Il est intéressant de noter que lorsque les petites exploitations sont plus nombreuses, les taux de participation des femmes agricultrices à l'emploi non agricole tendent à être inférieurs. Par exemple, 57 \% des femmes agricultrices ont un emploi non agricole dans la région de production Southern Seaboard, et $62 \%$ dans la région Northern crescent.

\section{Revenus d'activité, avantages des salariés et patrimoine}

Globalement, la tendance à l'emploi non agricole parmi les femmes agricultrices, combinée au fait que la moitié environ des agriculteurs ont aussi des emplois non agricoles, a conduit à une hausse de la dépendance des ménages agricoles envers les revenus non agricoles (d'activités et autres). Cette question en plusieurs dimensions mérite attention.

- -Selon les données portant sur les États-Unis, la part du revenu non agricole dans le revenu total des entreprises-ménages agricoles a augmenté de façon croissante, malgré des variations annuelles importantes liées à la variabilité du revenu agricole. Cette hausse reflète en partie l'augmentation des revenus d'activités des femmes au cours des trois dernières décennies. Selon les résultats de l'enquête de 1980, Rosenfeld rapporte que les revenus d'emploi non agricole des femmes représentaient environ $40 \%$ de ceux des hommes (Rosenfeld, 1985). Dans l'enquête de 2001, les revenus non agricoles des femmes représentaient environ $65 \%$ de ceux des hommes agriculteurs.

- -Les ménages agricoles des États-Unis reçoivent souvent des avantages sociaux financés par leur employeur, qui rentrent pour une grande part dans leurs décisions en terme d'emploi (Jensen et Salant, 1985). L'enquête de 2001 montre que davantage d'hommes que de femmes ayant un emploi non agricole reçoivent des avantages financés par leur employeur. Parmi ceux qui travaillent en dehors de l'exploitation, $69 \%$ des hommes (39\% de tous les agriculteurs) et $59 \%$ des femmes employées ( $40 \%$ de toutes les agricultrices) 
bénéficient d'une assurance maladie au travers de leur employeur. De nombreux ménages agricoles aux États-Unis ont également accès à une pension de retraite au travers de l'employeur qui les salarie. Parmi les agriculteurs ayant une activité non agricole, $61 \%$ ont accès à une pension au travers de leur employeur contre $55 \%$ des femmes. Il semble également que de nombreux salariés reçoivent des avantages groupés- assurance santé, assurance-vie et pension de retraite par exemple.

- -Les différences de revenu entre ménages agricoles et non agricoles sont faibles ou négligeables, en grande partie du fait des deux tendances décrites ci-dessus, (OCDE, 2003) ${ }^{4}$ montre que dans la plupart des pays développés, les revenus totaux des ménages agricoles et non agricoles sont pratiquement semblables. C'est le cas aux États-Unis lorsqu'on compare les ménages de l'enquête de la population actuelle (Current Population Survey, CPS). La comparaison des avantages reçus par les ménages agricoles par rapport à la population générale montre les membres de la famille agricole qui travaillent en dehors de l'exploitation sont plus susceptibles d'avoir des emplois qui procurent ces avantages. Par exemple, dans la population générale, $52 \%$ des travailleurs adultes aux États-Unis contribuent à un dispositif de sécurité sociale maladie, $54 \%$ à une assurancevie et $48 \%$ à un dispositif de retraite (US. Department of Labor, 2002a) $)^{5}$. En outre, les ménages agricoles sont moins imposés que les autres ménages car ils ont un traitement préférentiel (OCDE, 2003). Il est difficile de comparer le niveau du patrimoine car peu de sources de données comprennent les actifs non agricoles (OCDE, 2003).

\section{Tâches agricoles et prise de décision}

30 L'analyse des données de 2001 montre que de nombreuses femmes agricultrices aux ÉtatsUnis continuent de se considérer comme des participantes importantes aux activités de la ferme. En 1980 tout comme en 2001, un tout petit peu plus de la moitié des femmes agricultrices aux États-Unis se considéraient comme « l'un des principaux opérateurs ou le principal opérateur " de l'exploitation. Cependant, il y a eu des changements dans la participation des femmes agricultrices aux différentes formes de travail (tâches) sur la ferme ainsi que dans la prise de décisions qui lui sont liées. Entre 1980 et 2001, les données d'enquêtes montrent qu'il y a eu une hausse notable de la participation des femmes agricultrices aux travaux des champs et aux achats importants pour l'exploitation en fournitures et équipements (tableau 1). Des baisses ont également été enregistrées concernant : les soins aux animaux (66 à $59 \%$ ), l'entretien du jardin potager ou des animaux destinés à l'autoconsommation (88 à $74 \%$ ), la supervision du travail des salariés agricoles ( 36 à $28 \%$ ) et de la main d'œuvre familiale (49 à $36 \%$ ) sur l'exploitation, ainsi que le labour, le hersage, la culture ou l'ensemencement (37 à $33 \%$ ).

Comme elles se sont tournées vers l'emploi non agricole (emploi formel), les femmes agricultrices aux États-Unis semblent moins enclines à consacrer leur temps à des productions agricoles destinées à l'autoconsommation - prendre soin des animaux, cultiver un potager et se consacrer à d'autres tâches semblables qui appartiennent à la sphère élargie du ménage. Les femmes agricultrices en 2001 semblent aussi être moins enclines à s'engager dans la supervision des travaux agricoles effectués par la maind'œuvre familiale ou salariée. Compte tenu du vieillissement de la population agricole et de la diminution de la taille des familles en 2001 par rapport aux décennies précédentes, il n'est pas surprenant que les femmes agricultrices en 2001 aient moins tendance à s'engager dans la supervision des membres du ménage agricole (traditionnellement des 
enfants) lors de leurs activités agricoles. Le fait que les femmes agricultrices aient également moins tendance à superviser le travail des salariés agricoles, reflète peut-être la transition observée tout au long des années 1990 vers des salariés masculins, jeunes, célibataires, et d'origine hispanique dans l'agriculture des États-Unis (Mines, 2002). Dans le passé, la main d'œuvre salariée avait tendance à comprendre davantage de familles entières (Mines, op. cit.).

Tableau 1. Évolution de la participation des femmes agricultrices au travail agricole entre 1980 et 2001 aux Etats-Unis* (en \%)

\begin{tabular}{|l|l|l|}
\hline Tâche agricole & $\mathbf{1 9 8 0}^{* *}$ & $\mathbf{2 0 0 1}$ \\
\hline Labourer, herser, cultiver ou planter & 37 & 33 \\
\hline Épandre des engrais, herbicides et insecticides & 17 & 20 \\
\hline Travaux des champs & 42 & 48 \\
\hline Récolter, conduire des camions ou des machines agricoles & 51 & 50 \\
\hline Prendre soin des animaux de la ferme & 66 & 59 \\
\hline Faire des courses liées à l'exploitation agricole & 85 & 86 \\
\hline Achats importants d'intrant ou d'équipements agricoles & 37 & 48 \\
\hline Commercialiser les produits & 33 & 34 \\
\hline \hline Comptabilité & 78 & 78 \\
\hline Superviser les travaux d'autres membres de la famille & 49 & 36 \\
\hline Superviser les travaux de salariés agricoles & 36 & 28 \\
\hline S'occuper du potager ou d'animaux d'autoconsommation & 88 & 74 \\
\hline
\end{tabular}

* D'après des comparaisons (non publiées) réalisées par F. Willits et N. Jolly. Les réponses incluent les tâches régulières et occasionnelles.

** D'après les données fournies aux chercheurs par Rachel Rosenfeld.

La participation des femmes agricultrices aux grandes décisions concernant l'exploitation représente une autre dimension de leur engagement dans le fonctionnement de l'exploitation agricole. La comparaison des résultats de l'enquête de 2001 avec celle de 1980 montre que l'engagement des femmes dans la prise de décisions importantes a nettement changé (tableau 2). Presque la moitié des femmes agricultrices en 2001 ont répondu qu'elles étaient impliquées dans une gamme complète de décisions importantes concernant l'exploitation comme les achats et ventes de terres (73\%), la location de plus ou moins de terres (65\%), l'achat de d'équipements agricoles importants (58\%), l'achat de nouveautés comme une nouvelle culture ou une nouvelle race d'animaux (53\%), l'adoption d'une nouvelle méthode de production (53\%) et la date de vente des produits agricoles (49\%). Comme le montre le tableau 2, l'engagement des femmes agricultrices à 
la prise de décisions concernant l'exploitation a augmenté dans le cas de pratiquement toutes les décisions importantes.

Tableau 2. Participation des femmes agricultrices aux décisions concernant l'exploitation agricole aux États-Unis* (en \%)

\begin{tabular}{|l|l|l|}
\hline Décision & $\mathbf{1 9 8 0}$ & 2001 \\
\hline Acheter ou vendre des terres & 61 & 73 \\
\hline Louer plus ou moins de terres & 52 & 65 \\
\hline Acheter des équipements agricoles importants & 48 & 58 \\
\hline \hline Produire quelque chose de nouveau, & & \\
\hline (ex. nouvelle culture/ nouvelle race d'animaux) & 42 & 53 \\
\hline Essayer une nouvelle méthode de production & 37 & 53 \\
\hline A quelle date vendre les produits agricoles & 40 & 49 \\
\hline
\end{tabular}

* D'après des comparaisons (non publiées) réalisées par F. Willits et N. Jolly. Participation seule ou conjointe.

\section{Incidences sur les interactions au sein du ménage}

\section{Participation des femmes à l'emploi non agricole}

33 Les modèles de ménages peuvent être utilisés pour analyser les déterminants de la participation à l'emploi non agricole des femmes agricultrices, y compris pour identifier l'effet éventuel de la transmission intergénérationnelle de l'exploitation sur les décisions en matière l'emploi. Afin d'évaluer ces déterminants, des modèles probit bivariés ont tout d'abord été estimés pour établir la mesure dans laquelle, de nos jours, les décisions en matière d'emploi sont prises conjointement dans les exploitations des États-Unis. Un modèle global utilisant l'ensemble des données portant sur tous les couples d'agriculteurs a d'abord été estimé et la corrélation entre les équations (c'est-à-dire le coefficient rho) s'est révélée extrêmement significative au plan statistique dans les données de 2001 . Lundberg (1988) a avancé que les décisions des couples en matière l'emploi tendent à être prises en commun en présence d'enfants dans le ménage, mais séparément en l'absence d'enfants. Ces relations ont été testées pour des couples d'agriculteurs aux États-Unis mais aucune différence de "jointure » n'est apparu selon que le couple a des enfants ou non - dans les deux cas (avec ou sans enfant), les décisions en matière d'emploi non agricole des couples d'agriculteurs se révèlent corrélées.

À partir des résultats bivariés, ont été estimés des modèles logit multinomiaux qui identifient les effets de la transmission intergénérationnelle de l'exploitation ainsi que des caractéristiques des individus, du ménage, des exploitations et de leur situation régionale sur les décisions en matière l'emploi. Les résultats sont présentés dans les 
tableaux 3 et $4^{6}$. Deux modèles ont été estimés pour les femmes agricultrices - un pour celles dont le conjoint travaille sur l'exploitation à temps complet (selon le concept actuel d'agriculteur à temps complet') et l'autre pour les cas où il travaille en dehors de l'exploitation (et probablement sur l'exploitation également). En outre, les modèles se limitent aux individus en âge de travailler (de 18 à 64 ans inclus).

\section{Lorsque le conjoint n'est pas employé en dehors de l'exploitation} transmission de l'exploitation semblent moins importantes pour les décisions en matière d'emploi (tableau 4). Que l'exploitation soit transmise par la famille de l'un ou de l'autre conjoint ne semble pas affecter de manière importante les décisions en matière d'emploi. Cependant, l'âge de la femme affecte sa propension à travailler sur l'exploitation, uniquement ou en combinaison avec d'autres emplois - sa tendance à la pluriactivité augmente avec l'âge jusqu'à environ 38 ans, puis elle diminue. Cela semble être l'inverse pour son engagement dans le travail agricole (uniquement) : les femmes plus jeunes se considéreraient davantage engagées sérieusement dans l'exploitation alors que les femmes d'âge moyen, seraient plutôt moins impliquées. Cependant, après 41 ans, leur participation augmente de nouveau. Il convient de noter que cela correspond assez bien à l'âge auquel leur pluriactivité diminue.

Les effets marginaux estimés dans ce cas sont présentés au tableau 3. La probabilité que la femme agricultrice soit fortement impliquée dans les activités agricoles et n'ait pas d'emploi non agricole augmente avec la taille du ménage, lorsque son nom est inscrit sur le registre de l'exploitation, et si l'exploitation est située dans les régions Southern Seaboard, Fruitful Rim et Mississippi Portal. La probabilité qu'elle travaille exclusivement sur l'exploitation diminue plus son niveau d'éducation est élevé et plus les enfants du ménage sont âgés. Il est intéressant de noter que si l'exploitation provient de sa famille, elle est moins susceptible d'y travailler exclusivement lorsque son conjoint est déjà employé à temps complet dans l'agriculture.

l'exploitation ont uniquement un emploi non agricole et déclarent être peu impliquées dans l'activité agricole. La femme agricultrice est moins susceptible de travailler uniquement en dehors de l'exploitation si elle a été élevée sur une ferme, si le ménage compte beaucoup de membres et si son nom est inscrit sur le registre de l'exploitation. C'est également le cas si l'exploitation provient de la famille de son conjoint et si l'exploitation est située dans les régions Northern Great Plains et Eastern Uplands.

si elle a un niveau d'éducation élevé (à l'exception des diplômes agricoles), si elle a été élevée sur une ferme ou à la campagne (plutôt que dans une zone urbaine), et si les enfants ont atteint un certain âge. C'est également le cas si l'exploitation vient de sa famille ou de celle de son conjoint, plutôt que si elle a été achetée ou donnée et lorsque l'exploitation est située dans les régions Fruitful Rim ou Basin and Range.

\section{Lorsque le conjoint est employé en dehors de l'exploitation}

Avoir grandi dans une ferme tend également à influencer les choix des femmes en matière d'emploi lorsque leur conjoint travaille en dehors de l'exploitation. Par rapport aux femmes originaires de milieux urbains, les femmes originaires du milieu agricole 
répondront plus probablement qu'elles sont pluriactives et se considéreront plus rarement comme employées agricoles uniquement. Les femmes dans des ménages de plus grande taille seront plus probablement à temps complet sur l'exploitation et travailleront moins fréquemment en dehors de l'exploitation. Les femmes dont le nom est inscrit sur le registre de l'exploitation travailleront moins fréquemment en dehors de l'exploitation, tandis que les femmes sur des exploitations spécialisées dans les grandes cultures auront davantage tendance à la pluriactivité sur et en dehors de l'exploitation et auront moins tendance à être engagées uniquement sur l'exploitation. Enfin, lorsque l'agriculteur travaille en dehors de l'exploitation, sa femme/compagne est moins susceptible d'avoir un seul emploi non agricole dans les régions Southern Seaboard, Fruitful Rim ou Basin and Range.

En comparant les résultats des deux tableaux, on voit que lorsque l'agriculteur ne travaille pas en dehors de l'exploitation et se consacre entièrement au travail agricole, l'engagement de sa femme dans des activités multiples (plutôt que seulement agricoles) est influencée positivement par son niveau de capital humain (dans ce cas, son éducation formelle) mais pas par son âge. En effet, le niveau d'éducation des femmes agricultrices considérées comme travaillant à temps complet sur l'exploitation (selon la définition correspondant à l'emploi du conjoint) sert à différencier les femmes capables de contribuer aux revenus d'activités non agricoles du ménage agricole de celles qui ne le sont pas. Cela ne semble pas être le cas lorsque l'agriculteur travaille en dehors de l'exploitation, auquel cas l'âge est une variable plus importante pour différencier les choix des femmes agricultrices en matière d'emploi. Les différences de niveau d'éducation semblent moins importantes.

41 La transmission intergénérationnelle de l'exploitation, qu'elle ait la famille de l'homme ou de la femme pour origine, semble affecter les décisions des femmes agricultrices en matière d'emploi si l'exploitation est ce qu'on appelle à temps complet. Les coefficients estimés pour les variables de transmission intergénérationnelle suggèrent que lorsque l'homme consacre tout son temps à l'activité agricole, sa femme/compagne est plus susceptible d'être pluriactive, quel que soit l'origine de l'exploitation. Elle considère que son rôle est de contribuer à la fois à l'emploi non agricole et d'être très engagée sur l'exploitation.

Les variables de situation régionale qui reflètent le type de production agricole semblent influer sur les choix en matière d'emploi que font les femmes agricultrices aux États-Unis. Par rapport à celles de la région du Heartland, les femmes qui vivent dans les régions Southern Seaboard, Fruitful Rim ou Mississippi Portal (c'est-à-dire dans la plupart du sud et de la côte ouest) sont plus susceptibles de se limiter à une activité agricole (sans emploi non agricole) si leur conjoint travaille à temps complet dans l'agriculture.

Enfin, plus le ménage compte de membres et plus la probabilité que les femmes agricultrices travaillent est faible en général, que le conjoint/compagnon travaille en dehors de l'exploitation ou pas. En outre, l'âge des enfants semble influencer grandement les décisions en matière d'emploi, principalement pour les femmes dont le conjoint est employé à temps complet sur l'exploitation, les femmes ayant des enfants plus grands étant plus fréquemment pluriactives sur et en-dehors de l'exploitation. 


\section{Prise de décisions concernant l'exploitation}

44 La prise de décision concernant l'exploitation pourrait également être influencée par l'origine de la transmission de l'exploitation, ainsi que par les caractéristiques de l'exploitation et du ménage considérées dans les modèles estimés ci-dessus. Des modèles probit de participation à la prise de décision concernant l'exploitation ont été estimés pour sept types de décisions évaluées dans l'enquête de 2001 :

1. achat ou vente de terres,

2. location de plus ou moins de terres,

3. achat d'équipements agricoles importants,

4. adoption de productions nouvelles ou de nouvelles méthodes de production,

5. à quel moment vendre les produits agricole,

6. décisions relatives à l'embauche de salariés agricoles,

7. emprunt d'argent.

Des modèles séparés ont été estimés pour la participation des femmes agricultrices à chacune de ces décisions relatives à l'exploitation. Les modèles ont été estimés pour examiner si la transmission intergénérationnelle de l'exploitation affecte aussi ou non l'engagement des femmes agricultrices au processus de prise de décision.

Le tableau 5 résume les effets marginaux des variables de transmission intergénérationnelle ${ }^{7}$. Les résultats montrent que le fait que l'exploitation provienne de la famille de l'homme a un effet négatif sur la participation de son épouse/compagne à la prise de décisions concernant l'exploitation. C'est le cas pour tous les types de décisions : acheter ou vendre des terres, décision concernant la location de terres, achat ou non d'équipements agricoles importants, adoption de nouvelles activités ou de nouvelles méthodes de production, date à laquelle vendre les produits agricoles, l'embauche de salariés agricoles, décision d'emprunter ou non de l'argent. Les résultats montrent également que le fait que l'exploitation agricole soit originaire de la famille de la femme influence positivement sa participation à certaines décisions concernant l'exploitation mais pas toutes (tableau 5).

Tableau 5. Participation des femmes agricultrices aux décisions concernant l'exploitation agricole aux États-Unis* (en \%)

\begin{tabular}{|l|l|l|}
\hline Décision & Femme & Homme \\
\hline \hline Acheter ou vendre des terres & ps & -** \\
\hline Louer plus ou moins de terres & $+^{* *}$ & $-* *$ \\
\hline Acheter des équipements agricoles importants & $+^{*}$ & - -** \\
\hline Produire quelque chose de nouveau & & \\
\hline ou essayer une nouvelle méthode de production & $+^{*}$ & $-* * *$ \\
\hline A quelle date vendre les produits agricoles & ns & $-* * *$ \\
\hline
\end{tabular}




\begin{tabular}{|l|l|l|}
\hline Embaucher des salariés agricoles & $+^{* *}$ & $-*$ \\
\hline \hline Emprunter de l'argent & ps & $-* * *$ \\
\hline
\end{tabular}

ps : pas significatif au plan statistique.

*** = significatif à 0.01 ou mieux; ${ }^{*}=$ significatif à 0.05 ou mieux ; * = significatif à 0.10 ou mieux.

\section{Pour conclure}

$\mathrm{Au}$ cours des deux dernières décennies, la mesure dans laquelle les ménages agricoles aux États-Unis dépendent d'activités et de sources de revenu non agricoles a nettement évolué. Tandis que la participation des agriculteurs à des emplois non agricole progressait légèrement au cours de la période, la part des femmes agricultrices ayant une activité non agricole augmentait nettement. Au plan du ménage dans son ensemble, ces tendances ont entrainé aux États-Unis une hausse des revenus des ménages agricoles, une amélioration de l'accès aux avantages du salariat et une convergence entre les revenus moyens des ménages agricoles et ceux des autres ménages.

La tendance à la hausse de la part des femmes agricultrices aux États-Unis ayant 1) une activité non agricole, 2) classées comme chef d'exploitation et 3) héritant de l'exploitation de leur famille par transmission intergénérationnelle a une incidence sur le travail du ménage au sein de l'exploitation et sur les décisions relatives au futur de l'exploitation. Ces tendances entraînent maintenant un grand intérêt pour les effets internes au ménage. Ces travaux examinent la question des transmissions intergénérationnelles selon que l'exploitation vient de la famille de l'homme ou de celle de la femme - à l'aide d'un cadre faisant appel à la négociation au sein du ménage. L'origine de la transmission intergénérationnelle de l'exploitation agricole, selon le sexe de l'héritier, semble influer sur la répartition du travail au sein du ménage et sur la prise de décision concernant l'exploitation.

D'abord, on constate que l'origine de la transmission influe sur les décisions des agricultrices en termes de travail/emploi lorsque le conjoint/compagnon est employé à temps complet sur l'exploitation. Dans les exploitations provenant des parents ou de la famille de la femme, elle se considère comme davantage impliquée dans l'exploitation. Ainsi, même si elle travaille à l'extérieur, ce qui est souvent le cas, elle est davantage susceptible de pratiquer la pluriactivité sur et en dehors de l'exploitation. En outre, les capacités humaines de l'agricultrice semblent être déterminantes dans sa décision de travailler à temps complet sur l'exploitation ou de pratiquer la pluriactivité. Par ailleurs, lorsque le conjoint/compagnon travaille à temps partiel en dehors de l'exploitation, l'âge de la femme semble un facteur plus important, la pluriactivité suivant le cycle de vie traditionnel. Dans ce cas, les variables exogènes sont probablement négligeables au sens statistique.

Ensuite, les décisions concernant l'exploitation agricole semblent être influencées par l'origine de la transmission de l'exploitation, la participation de la femme agricultrice à la prise de décision étant renforcée si l'exploitation vient de sa famille alors qu'elle s'amenuise si l'exploitation vient de la famille de son conjoint/compagnon. Une plus grande participation aux décisions devrait influencer les résultats de ces décisions et donc refléter les préférences potentiellement assez diverses au sein du ménage. 


\section{BIBLIOGRAPHIE}

Becker G. A treatise on the family. Harvard University Press, Cambridge, 1991.

Chiaporri p. Rational household labor supply. Econometrica, 1988, n56.

Chiappori p. Collective labor supply and welfare. Journal of Political Economy, 1992, n¹00.

Doss C. Intrahousehold resource allocation in an uncertain environment: evidence from Ghana. Thèse de Ph. D de l'Université du Minnesota, 1996.

Errington A. Handing over the reins: a comparative study of intergenerational farm transfers in England, France, Canada and the USA. Contribution présentée à la réunion annuelle de l'Agricultural Economics Society, Aberystwyth, Royaume-Uni, avril 2002.

Findeis J., Swaminathan H. Multiple job-holding among U.S. farm women: off-farm work and on-farm decision-making using a bargaining approach. Contribution présentée à la réunion annuelle de l'American Agricultural Economics Association, Long Beach, CA, août 2002.

Jensen H., Salant p. The role of fringe benefits in operator off-farm labor supply. American Journal of Agricultural Economics, 1985, 67.

Keyzer M., Phimister E. Cooperative gains in agricultural succession. Contribution présentée à la conférence de l'Agricultural Economics Society, Plymouth, Royaume-Uni, avril 2003.

Kimhi A., Nachlieli N. Intergenerational succession on Israeli family farms. Journal of Agricultural Economics, 2001, 52(2).

Kooreman p., Wunderlink S. The economics of household behaviour. St. Martin's Press, New York, 1997.

Lundberg S. Labor supply of husbands and wives: a simultaneous equation approach. The Review of Economics and Statistics, may 1988.

Manser M., Brown M. Marriage and household decision-making: a bargaining approach. International Economic Review, 1980, 21(1).

McElroy M. The empirical content of Nash-bargained household behavior. Journal of Human Resources, 1990, 25(4).

McElroy M., Horney M. Nash-bargained household decisions: toward a generalization of the theory of demand. Economic Review, 1981, 22(2).

Mines R. Family settlement and technological change in labour-intensive US agriculture. In J. Findeis, A.Vandeman, J. Larson and J. Runyan (eds.), "Dynamics of hired farm labour: constraints and community responses”, CAB-International, UK, 2002.

OCDE. Le revenu des ménages agricoles dans les pays de l'OCDE : Questions et réponses. OCDE, Paris, 2003.

Quisumbing A., de La Briere B. Women's assets and intrahousehold allocation in rural Bangladesh. IFPRI, Washington DC, FCND, 2000, Discussion Paper $n^{\circ} 86$.

Quisumbing A. Household decisions, gender and development. IFPRI et The Johns Hopkins University Press, Washington et Baltimore, 2003. 
Pesquin C., Kimhi A., Kislev Y. Old age security and inter-generational transfer of family farms.

European Review of Agricultural Economics, 1999, 26(1).

Rosenfeld R. Farm women. Chapel Hill, North Carolina State Univ. Press, 1985.

Schultz T.-P. Women's role in the agricultural household: bargaining and human capital. New Haven: Economic Growth Center, Yale Univ., 1999, Center Discussion Paper nº 803.

Singh I., Squire L., Strauss J. Agricultural household models: extensions, applications and policy. The Johns Hopkins Press, Baltimore, 1986.

Swaminathan H., Findeis J. Access to credit and women's work decisions: an empirical study in rural Malawi. Journal of Population, 2003, 9(1).

Udry C., Hoddinott J., Alderman J., Haddad L. Gender differentials in farm productivity: implications for household efficiency and agricultural policy. 1995. Food Policy, 1995, 20(5).

US. Department of Agriculture. 2002 census of agriculture. Washington, DC: NASS/USDA, 2004.

\section{ANNEXES}

Les effets de la transmission intergénérationnelle de l'exploitation ainsi que les caractéristiques des individus, du ménage, des exploitations et de leur situation régionale sur les décisions en matière l'emploi.

Tableau 3. Participation aux activités agricoles des femmes agricultrices aux Etats-Unis. Le conjoint/compagnon n'a pas d'activité non agricole (en 2001)

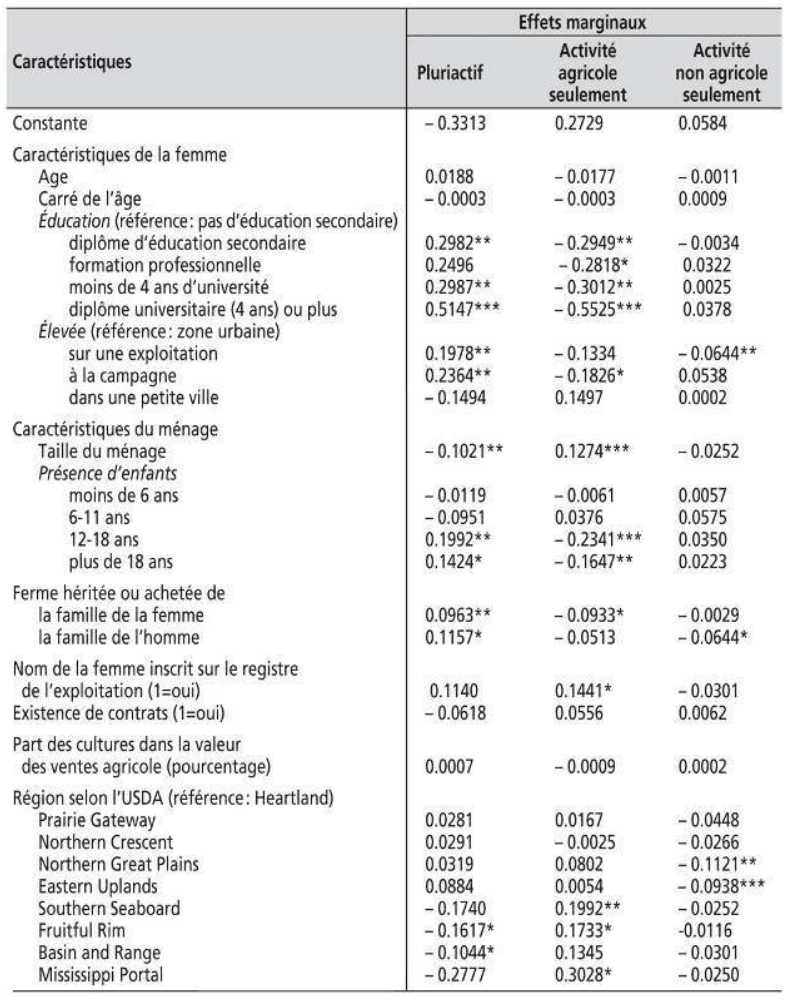

*** = significatif à 0.01 ou mieux; ** = significatif à 0.05 ou mieux ; * = significatif à 0.10 ou mieux. 
Tableau 4. Participation des agricultrices à l'emploi agricole aux Etats-Unis. Le conjoint a une activité non agricole (en 2001)

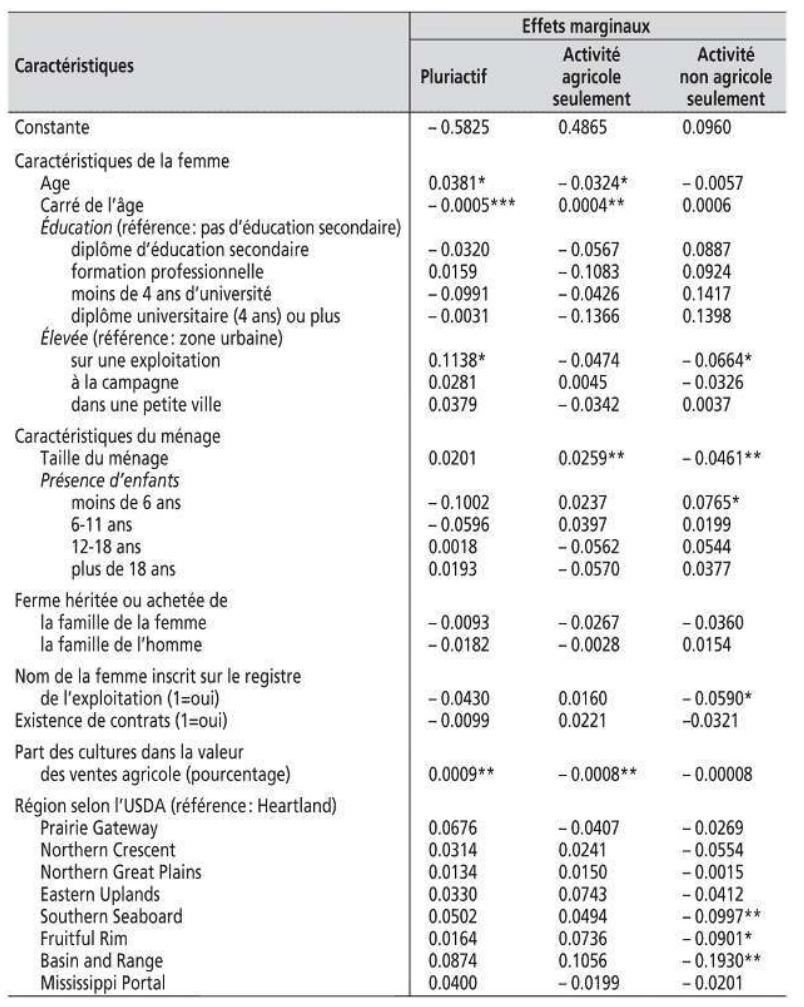

*** = significatif à 0.01 ou mieux; ** = signifıcatif à 0.05 ou mieux ; * = significatif à 0.10 ou mieux.

\section{NOTES}

1. L'intérêt que la société porte à l'agriculture durable et la préservation d'espaces ouverts dans les régions en développement a aussi contribué à ce renouvellement. OCDE, Impact de la réforme de la politique agricole sur l'emploi dans l'agriculture. OCDE, Paris, 2001, [AGR/CA/APR(2001)10/FINAL] 2. L'étude de Rosenfeld (Rosenfeld, 1985) a été menée en 1980, la participation à des activités non agricoles portant sur l'année précédente (1979). Dans l'étude de 2001, les données sur l'emploi correspondent également à l'année précédente (2000).

3. Dans les recensements de l'agriculture aux États-Unis, de celui 1974 au plus récent en 2002, ce taux varie d'un minimum de $55 \%$ (en 1974, 1992, et 2002) à un maximum de 58 \% en 1982 et 1997 (sans ajustement). Le recensement de l'agriculture de 2002 couvrait une population de ménages agricoles plus large que les recensements précédents.

4. Voir en particulier le graphique 1.1.

5. US, Department of Labor (2002a). News: Employment Characteristics of Families in 2002. Washington DC, Bureau of Labor Statistics, http://www.bls.gov/cps/

6. Les tableaux 3 et 4 sont exceptionnellement présentés en annexe.

7. Les modèles complets peuvent être obtenus auprès des auteurs. 


\section{RÉSUMÉS}

Dans les pays développés, les ménages agricoles sont hautement tributaires de la pluriactivité. L'emploi non agricole est très répandu et pour la plupart des ménages agricoles, son revenu représente une proportion non négligeable du revenu total. Selon les données d'une enquête nationale sur les agricultrices, menée à Penn State University en 2001, la participation des agricultrices à des emplois non agricoles a nettement augmenté au cours des dernières décennies. Pendant ce temps, le taux d'emploi non agricole des agriculteurs restait stable à $50 \%$. Les taux régionaux, en particulier pour les femmes, se sont montrés étonnamment élevés dans les régions d'agriculture traditionnelle et de faible densité. Depuis 1980, l'évolution des taux de participation à l'emploi non agricole s'est accompagnée d'une baisse de la participation des femmes aux tâches agricoles visant l'autoconsommation et d'une hausse nette et visible de la participation des femmes aux grandes décisions concernant l'exploitation. Étant donné l'évolution récente au sein des ménages agricoles, aux États-Unis comme dans de nombreux pays développés, il est admis que les interactions au sein du ménage sont probablement importantes. A l'aide d'une approche en termes de négociation dans le ménage, l'influence des types de transmission d'exploitations entre générations - selon que l'exploitation vient de la famille de l'homme ou de celle de la femme - est testée pour déterminer si le contrôle sur les actifs agricoles affecte la participation au travail agricole et non agricole et la prise de décision au sein de l'exploitation. L'intérêt de cette question vient de ce qu'il est maintenant de plus en plus fréquent aux États-Unis que les agricultrices héritent d'une exploitation. Les résultats montrent que si l'exploitation provient de la famille de l'agriculteur, celui-ci est plus susceptible de travailler sur l'exploitation et moins intéressé par des activités non agricoles, tandis que sa femme/compagne sera moins susceptible de travailler sur l'exploitation. A l'inverse, si l'exploitation vient de la famille de l'agricultrice, elle sera plus susceptible de travailler en dehors de l'exploitation, peut-être pour apporter un soutien financier permettant de poursuivre l'activité agricole. De forts effets sur la prise de décision sont observés: les agricultrices héritières sont beaucoup plus impliquées dans la prise de décision alors que c'est l'inverse si l'exploitation vient de la famille de l'agriculteur.

Farm households in developed countries are highly dependent on multiple job-holding and dual job-holding, with off-farm work being common and off-farm income comprising a significant portion of the income of most farm households. Using data from a national survey of farm women conducted by Penn State University in 2001, participation in off-farm work by women is shown to increase significantly over recent decades while rates among farmers are shown to be relatively stable around 50 percent. Regional rates, and particularly those for farm women, are shown to be surprisingly high in those regions where traditional agriculture is practiced and where population density is low. Since 1980, changes in off-farm participation to labor rates are shown to have occurred concomitantly with decreases in farm women's participation in farm tasks oriented toward home consumption and with significant increases in farm women's engagement in major farm decision-making. Given recent changes within farm households in the U.S. as well as in many developed countries, it is recognized that intra-household interactions are likely to be important. Using a household bargaining approach, the influence of intergenerational farm transfer patterns - through his family or though hers - is tested to 
determine whether farm asset control affects off-farm and farm participation à la main d'oeuvre and farm decision-making. The reason for this focus is that farm women in the United-States are now more likely to be the recipients of intergenerational transfers of farms than in the past. Results show that if the farm is transferred through his family, he is more likely to work on the farm and less likely to work off-farm, while she is less likely to work on the farm. Conversely, if the farm is transferred through her family, she is more likely to work off-farm, perhaps to provide financial support to continue the farm operation. Strong effects are observed relative to decision-making, with transfer through her family positively influencing her involvement in farm decision-making and transfer through his family strongly influencing her exclusion from farm decision-making.

INDEX

Mots-clés : agricultrices, emploi non agricole, ménage agricole, modèle de négociation (bargaining), prise de décision

Keywords : bargaining model, decision-making, farm household, farm women, off-farm labor Code JEL J12 - Marriage; Marital Dissolution; Family Structure

\section{AUTEURS}

\section{JILL L. FINDEIS}

Department of Agricultural Economics and Rural Sociology and Population Research, Institute Pennsylvania State University, University Park, Pennsylvania, USA

\section{HEMA SWAMINATHAN}

Department of Agricultural Economics and Rural Sociology and Population Research, Institute Pennsylvania State University, University Park, Pennsylvania, USA

\section{ANUJA JAYARAMAN}

Department of Agricultural Economics and Rural Sociology and Population Research, Institute Pennsylvania State University, University Park, Pennsylvania, USA 